Results Cana reduced the lipid (such as TG, TC, and LDLC) accumulation in serum, thus decreased atherogenic index of plasma and arteriosclerosis index values. More importantly, Cana decreased the thickness of the vascular basement membrane, improved cardiac mitochondrial homeostasis, and relieved oxidative stress (e.g. regulation of ROS, SOD, GSH, and MDA levels). And, Cana reduced the circulating markers of inflammation (such as TNF $\alpha$, MCP-1, and IL-6). Myocardial injury was alleviated after Cana treatment with decreasing levels of serous cTn I (from $95 \mathrm{pg} / \mathrm{mL}$ to $75 \mathrm{pg} / \mathrm{mL}$ ) and sCD40L (from $120 \mathrm{pg}$ / $\mathrm{mL}$ to $90 \mathrm{pg} / \mathrm{mL}$ ). Thus, the cardiovascular abnormality was relieved by elevating the CD31 expression level and suppressing fibrosis and basement membrane thickening (from $0.6 \mu \mathrm{m}$ to $0.2 \mu \mathrm{m}$ ) in $\mathrm{T} 2 \mathrm{DM}$ mice. Interestingly, Cana administration increased the ratio of Firmicutes/Bacteroidetes (from 230\% to 98\%) and the relative abundance of Olsenella, Alistipes, and Alloprevotella, while decreasing the abundance of Helicobacter and Mucispirillum in mice with diabetic CVD.

Conclusions Cana treatment improved CVD by decreasing the risk of atherosclerosis and reducing the thickness of the vascular basement membrane. Importantly, Cana treatment significantly elevated myocardial mitochondria homeostasis, thus ameliorated the oxidative stress and inflammatory states. Moreover, Cana subtly altered microbiota composition in T2DM mice with CVD, which contributed to the improvement of CVD. Collectively, the improvements of myocardial mitochondrial and gut microbiota homeostasis, may represent an important mechanism underlying the cardiovascular benefits of Cana treatment.

\section{IDDF2021-ABS-0199 REVEALING MOLECULAR AND CELLULAR DISCRIMINANTS OF TYPE 2 DIABETES BY INTEGRATIVE ANALYSIS OF PANCREATIC SINGLE-CELL RNA-SEQ DATA}

${ }^{1}$ Tao Zeng*, ${ }^{2}$ Hui Tang, ${ }^{3}$ Xiangtian Yu, ${ }^{2}$ Rui Liu. ${ }^{1}$ CAS Key Laboratory of Computational Biology, Bio-Med Big Data Center, Shanghai Institute of Nutrition and Health, University of Chinese Academy of Sciences, Chinese Academy of Sciences, Shanghai, China; ${ }^{2}$ School of Mathematics, South China University of Technology, Guangzhou, China; ${ }^{3}$ Clinical Research Center, Shanghai Jiao Tong University Affiliated Sixth People's Hospital, Shanghai, China

\subsection{6/gutjnl-2021-IDDF.57}

Background Type 2 diabetes (T2D) cause the dysfunction of insulin produced in the pancreas by beta cell, which leads to the non-correct response in fat, liver, and muscle cells. However, we are still far from completely understanding the molecular and cellular discriminants for T2D on cell level.

Methods On collected and integrated single-cell RNA-Sequencing (scRNA-seq) data from 3 publicly available pancreas datasets, we have trained an interpretable deep neural network model, which can provide an efficient T2D classifier to estimate the cell states from normal or T2D individuals and selected the key genes. Next, these key genes were used as anchors in down-stream analysis. Then, the cell type, marker gene, gene activity in cells, and cell-cell communications were all extracted and used in estimating the cell-type proportions and cell-type specific differential expressions in individualmatched bulk sequencing data.

Results We trained and tested T2D classifier to predict the states of cells from multiple single-cell datasets, and acquired significantly high accuracy $(\mathrm{ACC}=0.9034)$ in independent validation. Among key genes ranked by our classifier, there were 4, 12, 63 differentially expressed genes between normal and T2D states detected in acinar, alpha and beta cells, suggesting the stronger signal of dysfunction of T2D-relevant beta cells than other types of cells. These genes are significantly correlated with metabolic processes relevant to abnormal metabolism of T2D, and mainly show loss of expression, activity, and signaling changes among cell-cell communication in T2D state. Especially, the analysis results suggest that the beta-cell heterogeneity identified in single-cell dataset may be smaller in T2D relative to a normal state; by contrast, the alpha cell shows an opposite effect.

Conclusions Along with the development of high-throughput single-cell sequencing technologies, we are now capable of investigating the T2D pathogenesis on each cell in addition to each individual. There are actually necessary requirements on investigating cellular heterogeneity underlying individual heterogeneity of T2D, for personalized diagnosis and prognosis of T2D.

\section{IDDF2021-ABS-0200 BACILLUS AMYLOLIQUEFACIENS COMBINED WITH RESISTANT STARCH TO AMELIORATE INTESTINAL INFLAMMATION}

Hailan Zhao*, Yao Peng, Jing Xu, Haoming Xu, Wenqi Huang, Jiaqi Wang, Xue Guo, Yong Zhang, Hongli Huang, Youlian Zhou, Yuqiang Nie. Department of Gastroenterology, Guangzhou Digestive Disease Center, Guangzhou First People's Hospital, School of Medicine, South China University of Technology, China

\subsection{6/gutjnl-2021-IDDF.58}

Background Bacillus amyloliquefaciens (BA) decomposes starch by producing alpha-amylase to produce short-chain fatty acids (SCFA). Resistant starch (RS) can be fermented by colonic microbiota and metabolized to SCFA containing acetic acid and butyric acid by which can ameliorate intestinal inflammation. In this study, dextran sulfate sodium (DSS)-induced colitis mouse model was administrated to assess the therapeutic effect of BA combined with RS in experimental colitis.

Methods 6-week-old male $\mathrm{BALB} / \mathrm{c}$ mice were given 3\% DSS drinking water for 1 week to induce acute colitis. Mice were fed with maintenance forage as control group. The content of RS was $465.692 \mathrm{~g} / \mathrm{kg}$, and the abundance of BA by gavage administration in mice was $1 * 10^{7} \mathrm{CFU}$ for 7 days. The mice were administered daily, including weight loss and disease activity index (DAI). The mice were sacrificed on the eighth day. We collect specimens, measure colon length, evaluate histopathologic score and detect the level of IL-1 $\beta$, IL- 6 , TNF- $\alpha$ and occludin of intestinal tissue by qPCR.

Results Weight loss and DAI were more effectively improved in the live BA combined with RS than administered RS alone. Colonic inflammation was significantly alleviated in the live BA combined with RS. The colon length and histopathologic score of live BA combined with RS intervention were more improved than that of the control $(p<0.001)$, including a smaller extent of intestinal epithelial damage and crypt structure disorder. The degree of inflammatory cell infiltration was lighter $(p=0.029)$. The expression of IL-1 $\beta \quad(p=0.008)$, IL-6 $(p=0.02)$ and TNF- $\alpha(p<0.001)$ were reduced and the expression of Occludin $(p=0.006)$ was increased than RS alone. Conclusions The intervention of BA combined with RS may ameliorate DSS-induced acute colitis and promote colon barrier recovery. 